\title{
PLANES Y ACCIONES DE LOS PROFESIONALES DE LA COMUNICACIÓN DE MUSEOS EN CHILE. PROBLEMAS Y VISIONES
}

\section{Cristian Antoine y Javiera Carmona}
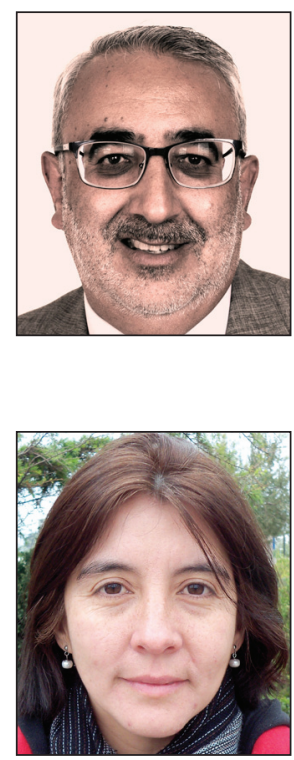

Cristian Antoine es doctor en ciencias de la información por la Universidad Complutense de Madrid (2002), periodista por la Pontificia Universidad Católica de Chile (1990) y profesor de historia y geografía en la Universidad de Chile (1985). Ex asesor del Gabinete del Ministro del Consejo Nacional de la Cultura y las Artes (2011), actualmente es director de Postgrado e Investigación de la Universidad del Pacífico, Chile.

http://orcid.org/0000-0001-8204-9630

Dirección de Postgrado e Investigación, Universidad del Pacífico Av. Las Condes, Casa Central 5o piso, 11121 Las Condes, Santiago, Chile cantoine@upacifico.cl

Javiera Carmona, periodista y magister en arqueología, cursó el doctorado en historia en la Universidad de Chile. Académica de la carrera de periodismo y los postgrados en arte, patrimonio y comunicación de la Universidad de Playa Ancha, Valparaíso. Su línea de investigación se centra en la comunicación, imagen y cultura, para abordar fenómenos diversos como la relación de los museos chilenos con sus audiencias, memoria de la catástrofe, y fotografía en Chile.

http://orcid.org/0000-0002-5519-416X

Universidad de Playa Ancha, Facultad de Ciencias Sociales Av. Playa Ancha 850, Casa Central 2o piso, Valparaíso, Chile javiera.carmona@upla.cl

\section{Resumen}

Se examina el concepto de comunicación museal desde la perspectiva del profesional a cargo de la comunicación del museo, caracterizando las circunstancias del ejercicio profesional y percepciones del área de comunicación. A través de una serie de entrevistas en profundidad con los responsables de la gestión de un conjunto de museos chilenos se identifican elementos a considerar y problemas que suelen afectar la comunicación de los museos en términos generales más allá de la diversidad que presentan estas instituciones. En los casos de los museos examinados se aprecia un escenario diverso en la concepción global del área de comunicación, que depende del emplazamiento geográfico, titularidad o dependencia, y de otras características del museo tales como misión, objetivos y visión de la comunicación.

\section{Palabras clave}

Museos, Comunicación, Profesionales de la comunicación, Departamento de comunicación, Chile.

Title: Plans and activities of communication professionals in Chilean museums. Problems and perspectives

\begin{abstract}
The concept of museum communication is examined from the perspective of the professionals in charge of this task, characterizing the practical circumstances, perceptions, and specifics of the communication area. Through a series of interviews with managers of a group of Chilean museums, we identified elements to consider and problems that often affect museum communications in general, despite the diversity of these institutions. In the museums examined, we observed different ways of understanding the concept of communication. Such differences are linked to geographical location, ownership or affiliation, as well as other dimensions of the museum such as mission, objectives of the institution, and the communications vision.
\end{abstract}

\section{Keywords}

Museums, Communication, Communication professionals, Communication department, Chile.

Antoine, Cristian; Carmona, Javiera (2014). "Planes y acciones de los profesionales de la comunicación de museos en Chile. Problemas y visiones". El profesional de la información, marzo-abril, v. 23, n. 2, pp. 165-172. 


\section{Introducción}

No hace mucho tiempo, desde esta misma revista Oliveira y Capriotti (2013) afirmaban que muchos museos se encuentran en una fase embrionaria de adopción de un nuevo modelo de gestión de sus públicos basado en la identificación de audiencias diferenciadas y estrategias de comunicación coherentes con esta diversidad. La incorporación de este paradigma innovador para abordar la difícil tarea de establecer relaciones eficientes y duraderas entre los museos y sus públicos plantea además algunas consideraciones sobre los profesionales que diseñan, ejecutan y finalmente evalúan los resultados de los planes implementados, dotados de ciertas capacidades y habilidades (Álvarez; Sebastián, 2010) para establecerse como "emitentes" o "emisores" del museo. Si bien es el museo el que "emite" mensajes y se "comunica", en la práctica quienes lo hacen son personas dotadas de ciertas concepciones e instrumentos técnicos dispuestos para ese fin y que ostentan un animus communicandi particular (Styliani et al., 2009).

Aunque es el museo el que 'emite' mensajes y se 'comunica', en la práctica quienes lo hacen son personas dotadas de concepciones e instrumentos técnicos dispuestos para este fin y que tienen un animus communicandi particular

Numerosos estudios de la comunicación museal han abordado la perspectiva del público receptor (Annis, 1994; Bialogorski; Cousillas, 2008; Bitgood; Thompson, 1987; Sanguinetti; Garré, 2001, entre otros), otros han centrado su atención en la perspectiva del mensaje emitido (Nardi, 2010; Styliani et al., 2009). Otra vertiente en crecimiento son los estudios de comunicación sobre el proceso de comunicación global del museo, que considera tanto los servicios de apoyo a la visita como los de mediación (MateosRusillo, 2008; 2012; Martín-Guglielmino; 2007).

En las tres tendencias señaladas, a menudo se soslaya de manera arbitraria la incidencia del experto comunicador en este proceso. Acometer tal ausencia es el objetivo del presente trabajo en el que se establecen las percepciones de los responsables de los departamentos de comunicación de los museos chilenos sobre su labor, a partir de las relaciones que establecen entre la misión y objetivos del museo, y el objetivo estratégico del área de comunicación. Asimismo, se identifica un conjunto de valores profesionales implícitos en la condición de comunicador museal que podría extrapolarse al colectivo profesional.

\section{La presencia del emitente en los estudios sobre comunicación museal}

Los museos han manifestado históricamente su preocupación por la conservación de la cultura y la comunicación de ésta. A juicio de Viñaras-Abad (2008), desde el siglo XVIII en adelante asumieron el primer tema, $y$ el segundo desde finales del siglo XX: comunicar el museo y su contenido con sus públicos. El reconocimiento de la necesidad de establecer vínculos con distintos públicos -de manera planificada, con personal cualificado- es reciente entre los responsables de los museos (Viñaras-Abad, 2005; 2008). Asimismo, se ha convertido en objeto de estudio formal para las ciencias de la comunicación (Alonso-Valero; Alonso-Valero; Caswell, 2003; Antinucci, 2004; Mateos-Rusillo, 2012) de manera que los precedentes de la evolución de este campo de estudio son amplios y bien conocidos desde $2000^{1}$, pero la aproximación al emisor, es decir, el componente "quién dice" (los profesionales que trabajan en el interior del sistema de comunicación), es un aspecto postergado en la discusión.

Según Viñaras-Abad (2005) la incorporación de comunicadores a estas instituciones culturales ha sido un proceso complejo y no exento de contradicciones debido al protagonismo histórico de la colección y su conservación, así como de los actores vinculados directamente con tal aspecto: el comisario artístico o curador ${ }^{2}$, conservador y/o director. En los últimos años, los encargados de la comunicación del museo con los públicos han ido adquiriendo mayor visibilidad hasta disputar el protagonismo con otros especialistas de la institución cuando se establecen consensos sobre el papel de este profesional en los procesos de comunicación museal (Alonso-Valero; Alonso-Valero; Caswell, 2003; Antinucci, 2004). La comprensión de la visita como "experiencia museal" se ha documentado en las acciones sobre el sistema de comunicación del Museo Reina Sofía en Madrid (Martínez-Peláez; Oliva-Marañón; Rodríguez-Rivas, 2012), y del Museo Altamira (Cantabria) basadas en la adopción de la noción de "comunicación global" (Fatás-Monforte, 2004). Sin embargo, son escasas las referencias de los museos latinoamericanos en esta dirección (Cóppola; González, 2009; De-las-Nieves-Sarno; Grandi; Lloret, 2006).

El organismo encargado de planificar y llevar a cabo la estrategia de comunicación del museo es el Departamento de Comunicación, en el que se plantean las estrategias de comunicación teniendo en cuenta especialmente - pero no únicamente- la vinculación con los públicos (Álvarez; Sebastián, 2010). De hecho, la difusión del patrimonio se gestiona diseñando estrategias específicas para cada proyecto a partir de los públicos a los que va dirigido, o teniendo en cuenta aquellos a los que no va dirigido (Saavedra-Cruz, 2013). Se considera que la eficacia de una estrategia de vinculación con el entorno dependerá:

- de la comprensión por los profesionales de la comunicación del papel del museo en la mediación cultural que emprende (Nardi, 2010);

- de las competencias y habilidades de los responsables técnicos de llevar adelante estos procesos;

- de los medios y recursos disponibles en los departamentos de comunicación y/o difusión para lograrlo.

\section{Metodología: entrevistas en profundidad}

La muestra para las entrevistas en profundidad se determinó a partir de la base de datos de los museos chilenos realizada en 2012 por nuestro grupo de investigación. El análisis con el programa estadístico SPSS Statistics de IBM se hizo considerando como variables de selección de la muestra la "audiencia durante el último año", categorizada luego en 5 tipos de respuestas (variable dependiente) (tabla 1). 
Tabla 1. Variable "audiencia durante el último año": cantidad de visitantes estimada

\begin{tabular}{|l|}
\hline 1: $0-10.000$ \\
\hline 2: $10.001-20.000$ \\
\hline 3: $20.001-30.000$ \\
\hline 4: $30.001-40.000$ \\
\hline 5: más de 40.000 visitantes \\
\hline
\end{tabular}

Las variables independientes se relacionaron con los medios de comunicación que pudiesen utilizar los museos ${ }^{3}$ (tabla 2).

Tabla 2. Medios de comunicación usados por los museos

\begin{tabular}{|c|l|c|l|}
\hline 1 & Web propia & 4 & Cuenta en Twitter \\
\hline 2 & E-mail para contacto & 5 & Cuenta en Facebook \\
\hline 3 & Redes sociales digitales & 6 & Medio de comunicación propio \\
\hline
\end{tabular}

A los encargados del área de comunicación de los museos seleccionados (tabla 3 ) se les entrevistó en su propio museo, entre mayo y agosto de 2013. Las entrevistas duraron un promedio de 1 hora, se grabó en audio la conversación y luego fue transcrita de manera íntegra para realizar el análisis. La entrevista en profundidad es recomendada por Ruiz y Aramburo (2012) sobre el resto de recursos metodológicos disponibles para realizar investigaciones en museos. Meluch (2010) la integra además entre los recursos para el conocimiento eficiente de las audiencias del museo. La adopción del enfoque cualitativo ${ }^{4}$ requirió el uso del programa Atlas.ti 6,0 para el análisis de las entrevistas.

El análisis de las entrevistas se inició a partir de dos grandes categorías preliminares: 1) Plan de comunicaciones, y 2) Área de comunicación. La primera abarcó 4 subcategorías y la segunda 6 (tabla 4) bajo las que se agruparon los temas que surgieron del diálogo con los entrevistados.

\section{Resultados}

\section{Visión, misión, objetivos, funciones}

Cinco de los nueve museos señalan tener una misión establecida, entendida como el propósito general de la institu- ción, y corresponden a los estatales y los de grandes instituciones (universidades, fuerzas armadas). Las misiones señaladas incluyen investigar, conservar, difundir y educar. Tres de ellos declaran favorecer la valoración y protección del patrimonio por medio de su conservación y difusión; de este modo el eje de su misión privilegia los bienes culturales que custodia sobre cualquier otro actor relacionado con el museo (como la comunidad). Llama la atención que ninguno declara disponer de una visión de la institución, es decir, una mirada a largo plazo que sitúe al museo en el futuro a partir del reconocimiento de las condiciones cambiantes del entorno en el que opera; una suerte de "presentismo" guía o dirige entonces las acciones de comunicación.

A excepción de dos museos (Nacional de Historia Natural y Mapuche de Pucón), el resto declara la intención de acercar la comunidad al museo y hacer más accesible la información o el conocimiento específico que éste posee; ambos son considerados los objetivos principales de la institución. Se expresa en ideas como:

- hacer más accesibles y cercanos los materiales y temas que se exhiben en el museo (Historia Natural de Valparaíso);

- ser una ventana para dar a conocer, abrirse a la comunidad (Geológico Lajos Biró Bagoczky);

- acercamiento a la comunidad (Histórico de Carabineros);

- que la gente conozca su historia (Isla de Maipo).

En algunos casos este objetivo se relaciona con fomentar la valoración del patrimonio cultural (Arqueológico de La Serena, Fonck e Isla de Maipo) -algo próximo a la noción de misión-, lo cual es de difícil verificación en su cumplimiento o comprobación, así como de expresar de manera más precisa acciones de las que derivan resultados. Este objetivo señalado por los entrevistados expresa la vigencia de la tradicional función de conservación y defensa de los bienes culturales, propósito privilegiado por los museos locales. Este rasgo es el antecedente que originó el marco legal desde el que se fundaron los museos provinciales o regionales en Argentina (Caballero-Zoreda, 1980; p. 378) y en Chile.

La complejidad del museo está en que articula varias funciones y cada una apela a profesionales con formaciones específicas que despliegan sus cometidos en coherencia con una

Tabla 3. Numeración de museos-fuente

\begin{tabular}{|c|c|c|c|c|}
\hline $\mathbf{N}^{\circ}$ & Museo & Dependencia & Ubicación & Fundación \\
\hline P 1 & Historia Natural de Valparaíso & Dibam & Valparaíso & 1878 \\
\hline P 2 & Geológico Lajos Biró Bagoczky & Universidad de Concepción & Concepción & 2003 \\
\hline P 3 & Gustavo Le Paige & Universidad Católica del Norte & San Pedro de Atacama & 1963 \\
\hline P 4 & Histórico de Carabineros & Carabineros de Chile & Santiago & 2009 \\
\hline P 5 & Nacional de Historia Natural de Santiago & Dibam & Santiago & 1830 \\
\hline P 6 & Arqueológico de La Serena & Dibam & La Serena & 1946 \\
\hline P 7 & Museo de Arqueología e Historia Francisco Fonck & Particular (Familia Fonck) & Viña del Mar & 1937 \\
\hline P 8 & Mapuche de Pucón & Particular (Familia Ulloa Metzger) & Pucón & $\sin$ fecha \\
\hline P9 & Museo del Centro Cultural Isla de Maipo & Centro Cultural Nuestra Señora de la Merced & Isla de Maipo & sin fecha \\
\hline
\end{tabular}


misión sobre el sentido último de la institución. Los inconvenientes para distinguir entre funciones concretas y misión del museo, como declaración del uso social de la institución (fortalecer la identidad local, desarrollar un proyecto educativo, fomentar el turismo cultural, etc.), constituyen escollos para precisar no sólo el perfil de los profesionales a cargo de ejecutar tales funciones, sino que también dificultan la elaboración de un plan estratégico de comunicación coherente con la misión del museo. En esta línea, Berta Sureda, directora de Actividades Públicas del Museo Reina Sofía sostiene que "Si anteponemos el museo como lugar de conocimiento y educación, donde se interpela al visitante y se le ofrecen herramientas para que construya sus propios relatos, estamos cumpliendo nuestra misión. Desviarse de nuestras líneas estratégicas o misión, cegados por la captación de más y más público, no consolidará el proyecto con sus propias especificidades" (Martínez-Peláez; Oliva-Marañón; Rodríguez-Rivas, 2012, p. 5). La incorporación a la misión de los museos de la idea de situar en lugar privilegiado a la audiencia, reconociendo su amplitud y diversidad, como orientadora y destinataria fundamental de sus acciones, aclara la ruta para despejar y establecer su razón de ser.

\section{Objetivo área de comunicación, Objetivos del museo}

Los entrevistados que señalaron el acercamiento a la comunidad como objetivo de museo coinciden en situarlo también como el objetivo del área de comunicación. Las funciones $u$ objetivos atribuidos históricamente a los museos no han variado fundamentalmente, pero sí su orientación, de manera que "hoy se conserva, se investiga y se difunde para todos, con una clara voluntad comunicadora" (Fatás-Monforte, 2003, p. 131). Algunos museos señalan como objetivo del área de comunicación la realización de actividades especiales para atraer al público. Dos museos (Gustavo Le Paige y Fonck) descartan como objetivo explícito atraer audiencia; el foco estaría en la calidad de la muestra museográfica o la conservación, la llegada del público al museo es consecuen- cia de ello. La superposición entre los objetivos del museo y del área de comunicación, revela una concepción tácita del museo como un agente comunicador pero sin detenerse a examinar las competencias o capacidades comunicativas del museo en la interacción con sus destinatarios.

\section{Características del área de comunicación, inserción institucional y plan estratégico}

El área de comunicación varía entre los museos examinados según su consolidación en la institución, tamaño de los equipos técnicos y disponibilidad de recursos. Los museos estatales o de grandes instituciones (universitarias, fuerzas armadas) disponen de un área de comunicación mejor establecida (con profesionales a cargo, recursos asignados, diversidad de actividades). En cambio, el Museo Gustavo Le Paige asume la comunicación con sus audiencias en la propuesta museográfica; la relación comunicacional es "desde las puertas para adentro" del museo.

En cada museo se aprecia variedad en la ubicación y articulación del área de comunicación con el resto de las áreas. El papel de los encargados y las posibilidades de realizar actividades depende no sólo de sus propias capacidades de gestión, sino también de la valoración de los procesos comunicacionales y de vinculación con la audiencia en la propia institución museal. En tres casos (Histórico de Carabineros, Arqueológico de La Serena, Fonck) se señala la especificidad del área de comunicación y la realización de procesos o formas de trabajo especializadas en su interior, lo que ha permitido negociar con sus jefaturas o con otras áreas de la organización. El área de comunicación es un departamento reciente y los profesionales incorporados se encuentran elaborando un modelo de trabajo que implica una etapa fundacional del área, como en el caso del Museo Histórico de Carabineros. En los museos estatales del conjunto examinado, la creación del área responde a las orientaciones generales a nivel nacional de la Dirección de Bibliotecas, Archivos y Museos de Chile (Dibam) y consiste en la capacitación en

Tabla 4. Categorías y subcategorías preliminares para análisis de entrevistas

\begin{tabular}{|c|c|c|}
\hline Categorías & Subcategorías & Descripción \\
\hline \multirow{4}{*}{$\begin{array}{l}\text { Plan de } \\
\text { comunicación }\end{array}$} & Existencia plan estratégico de comunicación (PEC) & Tiene / no tiene \\
\hline & Objetivos área comunicación & Razones u objetivos para los cuales genera procesos comunicativos \\
\hline & Desarrollo e implementación plan de comunicación & $\begin{array}{l}\text { Cómo lo hicieron, lugar que ocupa en la gestión diaria del museo, } \\
\text { cómo se implementa y evalúa }\end{array}$ \\
\hline & Razones ausencia PEC & Por qué el museo no ha creado un plan estratégico de comunicación \\
\hline \multirow{6}{*}{$\begin{array}{l}\text { Área de } \\
\text { comunicación }\end{array}$} & Tareas cotidianas encargado comunicación & Tareas que son parte de la rutina del encargado de comunicación \\
\hline & Estrategias y actividades área comunicación & Principales actividades realizadas desde el área de comunicación \\
\hline & Obstáculos institucionales & $\begin{array}{l}\text { Situaciones o condiciones institucionales que restringen la función } \\
\text { del área de comunicación }\end{array}$ \\
\hline & Inserción institucional comunicación & Rol del área de comunicación en museos \\
\hline & Condiciones de la institución & $\begin{array}{l}\text { Características generales, situaciones particulares y dinámicas insti- } \\
\text { tucionales }\end{array}$ \\
\hline & Constitución de área de comunicación & Cuándo y cómo se creó el área de comunicación \\
\hline
\end{tabular}


comunicación del profesional del área de educación (Historia Natural de Valparaíso). Se aprecia la tendencia (no sólo en museos estatales) a percibir los departamentos de educación y comunicación cumpliendo la misma función, de manera que quedan fundidos en uno solo.

Sólo dos museos (Histórico de Carabineros y Nacional de Historia Natural) poseen planes estratégicos de comunicación. Un tercero lo tuvo sin ejecución porque el museo permanece cerrado por reformas (Arqueológico de

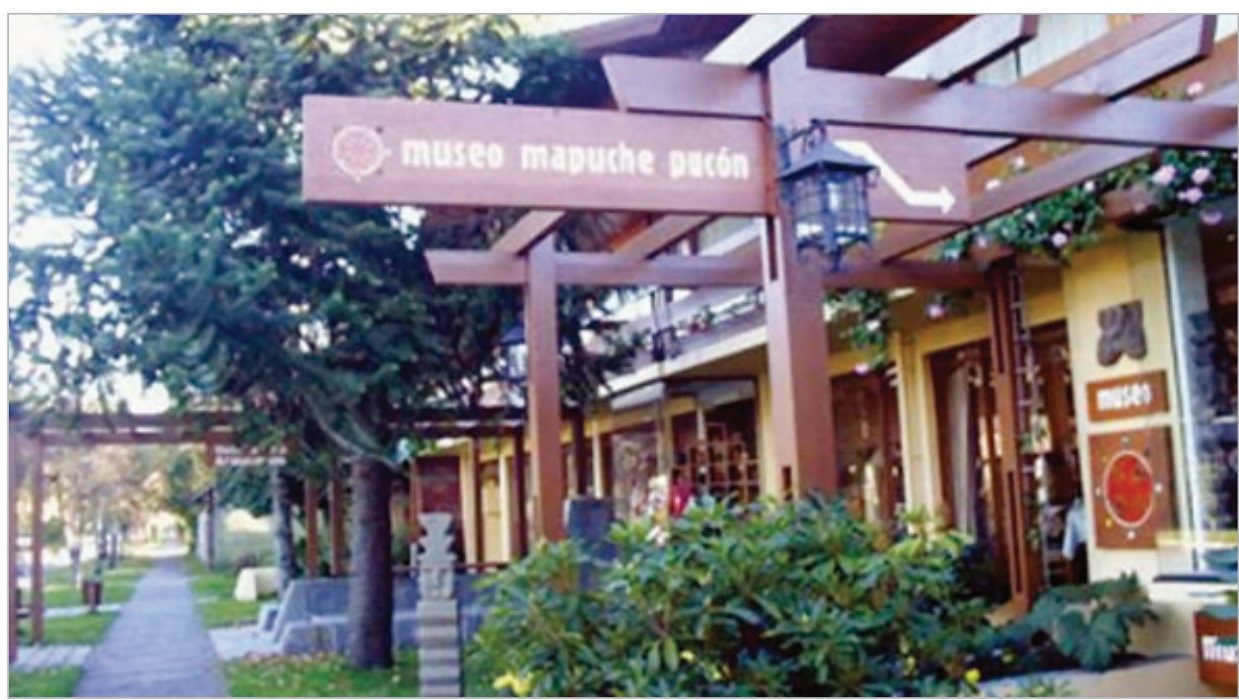

Museo Mapuche en Pucón La Serena). Las razones que los siete museos restantes esgrimen sobre la ausencia de un plan son, principalmente, la limitación de los recursos profesionales, que se expresa en conocimiento técnico específico insuficiente en los encargados (Historia Natural de Valparaíso); falta de personal y de recursos para comunicación (Geológico Lajos Biró Bagoczky, Gustavo Le Paige, Isla de Maipo); o tiempo escaso para los profesionales a cargo (Arqueológico de La Serena). También se señala que no exis-

te un interés específico del museo en esa función (Gustavo Le Paige e Isla de Maipo, de forma implícita).

\section{Estrategias y actividades del área de comunicación}

Son diversas y de intensidad variable las actividades de comunicación que cada museo realiza de acuerdo con el respaldo institucional y el desarrollo del área. Se detallan en la tabla 5 .

Tabla 5. Estrategias y actividades comunicacionales realizadas por los museos

\begin{tabular}{|c|c|}
\hline Estrategia & Actividades \\
\hline $\begin{array}{l}\text { Difundir las actividades y servicios del } \\
\text { museo desde el museo }\end{array}$ & $\begin{array}{l}\text { "Poner la cartelera", habitualmente en medios del propio museo como letreros en la entrada, envío } \\
\text { de correos electrónicos, publicación de notas informativas en la web del museo o en redes sociales } \\
\text { (Facebook y Twitter). }\end{array}$ \\
\hline $\begin{array}{l}\text { Difundir las actividades y servicios del } \\
\text { museo fuera del museo }\end{array}$ & $\begin{array}{l}\text { Realizan visitas y charlas a colegios. Crean dispositivos comunicacionales para ser utilizados en } \\
\text { escuelas y espacios de circulación de personas (salas de espera). Por ejemplo, el Museo Fonck está } \\
\text { elaborando un vídeo promocional. }\end{array}$ \\
\hline $\begin{array}{l}\text { Ofrecer el museo como atractivo } \\
\text { turístico }\end{array}$ & $\begin{array}{l}\text { Gestionar la aparición del museo en guías turísticas, incorporar la visita en los paquetes y circuitos } \\
\text { turísticos con los operadores y con la entidad gubernamental a cargo (Servicio Nacional de Turismo } \\
\text {-Sernatur). Esta acción es especialmente importante para los museos de lugares turísticos como San } \\
\text { Pedro de Atacama, Pucón y Valparaíso. }\end{array}$ \\
\hline $\begin{array}{l}\text { Generar noticias y difundir las activida- } \\
\text { des del museo en medios de comunica- } \\
\text { ción masivos ( } \mathrm{mcm} \text { ) }\end{array}$ & $\begin{array}{l}\text { Enviar comunicados de prensa a radios, diarios y canales cerrados de televisión (por ejemplo en el } \\
\text { Metro de Santiago). Se usa habitualmente para promover actividades especiales que resulten atracti- } \\
\text { vas para los medios de comunicación masivos. }\end{array}$ \\
\hline $\begin{array}{l}\text { Crear medios de comunicación propios } \\
\text { para la difusión del museo }\end{array}$ & $\begin{array}{l}\text { Elaboran revistas y boletines institucionales, así como catálogos, folletos, fichas. Por ejemplo, el Museo } \\
\text { Histórico de Carabineros edita una revista mensual e imprime catálogos y folletos. }\end{array}$ \\
\hline $\begin{array}{l}\text { Promover la interacción entre el museo y } \\
\text { los públicos }\end{array}$ & $\begin{array}{l}\text { Utilizan redes sociales y espacio en la web del museo para establecer interacción con el público. } \\
\text { Los museos del estado (Dibam) disponen de un sistema de recepción de quejas, reclamaciones, } \\
\text { observaciones y felicitaciones, de manera semejante a todas las reparticiones del estado en las que se } \\
\text { atienden usuarios. }\end{array}$ \\
\hline $\begin{array}{l}\text { Atraer grupos, colectivos y organizacio- } \\
\text { nes definidas }\end{array}$ & Realizan visitas guiadas especiales para colegios, empresas, organizaciones sociales, etc. \\
\hline $\begin{array}{l}\text { Realizar actividades específicas para } \\
\text { atraer ciertos públicos }\end{array}$ & $\begin{array}{l}\text { Ejecutan talleres y charlas. Por ejemplo: "Bueno y si traemos algo importante y organizamos actividades. } \\
\text { Dejemos un día anual para que los niños hagan manualidades, pinten y todo y tuvimos } 1.700 \text { personas en } \\
\text { un viernes" (12:3) }\end{array}$ \\
\hline
\end{tabular}




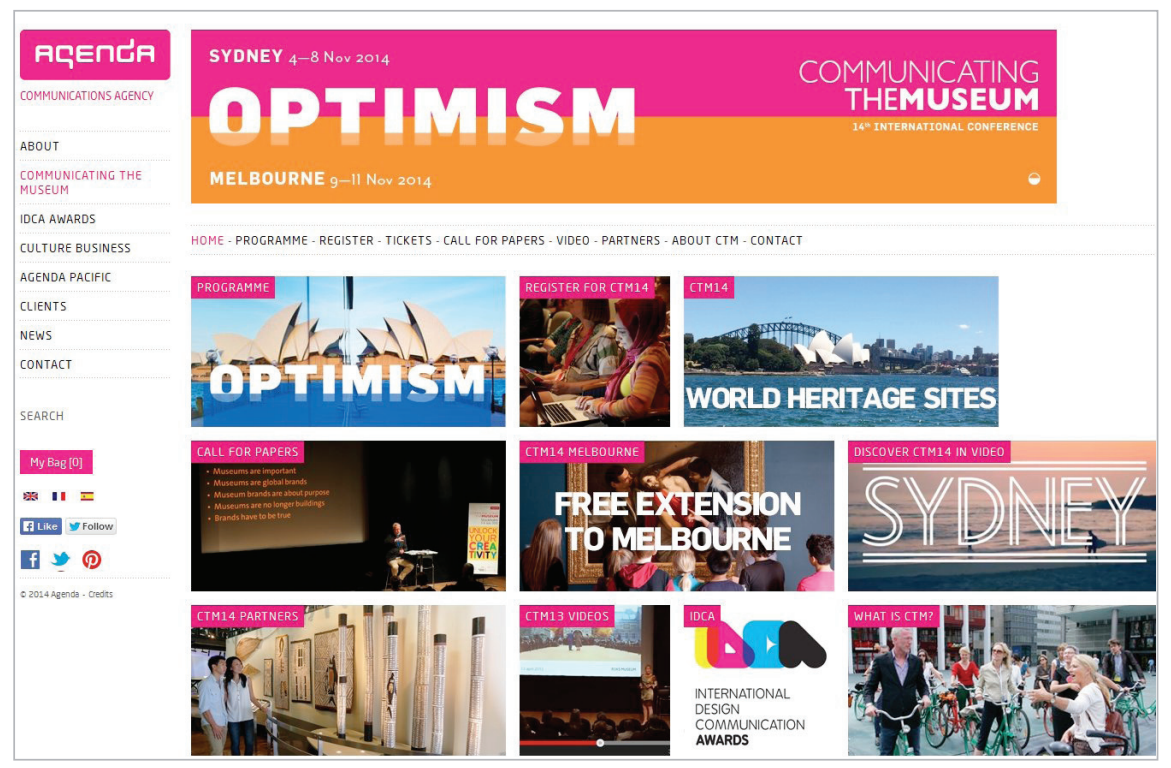

La 14a conferencia Communicating the museum tendrá lugar entre Sidney y Melbourne los días 4-11 de noviembre de 2014 , con el lema Optimism.

http://agendacom.com/en/communicating_the_museum

Algunos entrevistados destacan ciertas acciones como especialmente exitosas dentro de las estrategias, como potenciar la imagen corporativa (Nacional de Historia Natural). Es así como se explica que todas las acciones y actividades que emprende hacia el exterior sean interpretadas por los emitentes como parte de una estrategia de comunicación, aun cuando hay tareas que podrían situarse en otros ámbitos como educación, por ejemplo.

\section{Visión sobre comunicación}

El tipo de actividades se relaciona con las visiones de comunicación presentes en los museos, siendo la más extendida la de generar informaciones y difundirlas para que el público en general conozca lo que hace la institución. Algunos entrevistados reconocen lo limitante de esta visión y advierten que podrían realizar otras acciones, pero no saben bien cómo hacerlo o no tienen los recursos: "Para nosotros la difusión es más que nada hacer una venta de todos nuestros servicios digitales, hacer una cartelera de las actividades que tenemos y vender el servicio al público, pero no tenemos esta parte comunicacional, que es otro tipo de sistema, donde uno informa más en profundidad, genera noticias, genera otro tipo de expectativas para el público y no tenemos ese tipo de concepto desarrollado" (Historia Natural de Valparaíso).

Persiste también la visión sobre la efectividad de la transmisión informal entre los visitantes sobre el atractivo del museo, el "boca a oído", lo que estimularía el interés de visitarlo en otros públicos, especialmente en los museos regionales. "Que los chiquillos le cuenten a sus amigos, que se corra la voz, porque a pesar de todas las tecnologías yo soy una convencida de que (...) más allá de cualquier estrategia comunicacional, el boca a oído es lo más seguro" (Museo de Historia Natural de Valparaíso). Tal afirmación relativiza la importancia de una estrategia de comunicaciones para establecer una relación duradera y eficiente con los públicos y sitúa en un lugar privilegiado la experiencia museal. Concibe la exposición como medio de comunicación, en la que se articularía la museografía con los ya mencionados servicios de apoyo (información, orientación, descanso) y mediación (permiten la decodificación de la propuesta museal) que define Mateos-Rusillo (2008). Algunos entrevistados señalan lo conveniente de la comunicación bidireccional, de recoger de manera eficiente las opiniones de la audiencia, pero reconocen la ausencia de recursos o mecanismos que les permitan obtener y procesar la información de manera sistemática. Lo realizan de forma intermitente y asistemática, atendiendo a la interacción desde las redes sociales y en algunos casos a través de los comentarios en los libros de visitas.

\section{Discusión y proyecciones}

Hace más de 30 años Caballero-Zoreda (1980, p. 384) afirmó que "es necesario decir y explicar a la sociedad que las funciones del museo no están cubiertas", lo que se puede interpretar no sólo como el intento de convencer sobre la necesidad de incorporar al equipo de los museos a profesionales más allá de las "disciplinas del pasado" (arqueólogos, historiadores, historiadores del arte); tal es el caso de los comunicadores. También la frase de CaballeroZoreda se puede comprender como el esfuerzo por persuadir sobre la necesidad permanente del museo para la sociedad contemporánea porque aún no hay institución que pueda reemplazarlo en los objetivos que cumple. Visto así, la incorporación al museo de especialistas en comunicación responde a la reconsideración sustancial del perfil de los profesionales, facultados ahora en comunicar a la sociedad estas dos cuestiones fundamentales:

- la vigencia de la institución en la actualidad (misión);

- la complejidad de los objetivos que acomete (funciones de conservar, investigar, educar y difundir) sobre los bienes culturales que custodia con una orientación comunicativa (diversidad de públicos).

Los responsables de comunicación en los museos examinados revelan que el emplazamiento geográfico del museo y su titularidad intervienen con distinta intensidad en la concepción global del área de comunicación y la articulación de sus dimensiones (misión del museo, objetivos/funciones del museo, objetivos/funciones del área de comunicación, visión de la comunicación).

El Estado y las fuerzas armadas a través de sus museos son emisores que determinan la comprensión y valoración del proceso comunicativo, los recursos asignados y las acciones de sus encargados de comunicación. Incluso estos museos, localizados en la capital del país, señalan la importancia de crear y fortalecer una imagen-marca dentro de un plan estratégico de comunicación, lo que revela la percepción sobre la necesidad de posicionarse favorablemente en un conjunto de ofertas culturales provenientes de diversos agentes en competencia (cines, teatros, espectáculos musicales y deportivos, etc.). 
El escenario es muy dispar frente al resto de los encargados de comunicación de museos universitarios y privados, tanto en la percepción sobre su cualificación, oportunidades de desarrollo del área, efectividad de las acciones y por último, de la evolución del concepto de museo.

Los museos regionales/provinciales mantienen una visión de la comunicación más restringida que depende del "boca a oído" y la comprensión de la exhibición como único espacio comunicativo (privilegia el papel del comisario y su criterio ante la ausencia de profesionales de la comunicación). Además, exploran intuitivamente el uso de las redes sociales digitales, pero la relación con los medios de comunicación masivos es prácticamente inexistente. Esta visión compite con la mirada estratégica de la comunicación de los museos capitalinos/centrales.

\section{Notas}

1. Las propuestas oscilan entre una consideración amplia del museo concebido como "medio o espacio de comunicación" (Alonso-Valero; Alonso-Valero; Caswell, 2003; Annis, 1994; Antinucci, 2004); la labor del comunicador en el interior de la institución museal y las variables de comunicación corporativa que ella comporta (Caballero-Zoreda, 1986; FatásMonforte, 2004) y la condición de fuente de noticias que el museo y la exposición supone para los medios (JiménezLosantos, 2004; Lledó, 2003; Pulido, 2004), entre otros

2. En Chile y varios países latinoamericanos (México, Argentina, Perú) se utiliza la expresión "curador" para referirse al comisario artístico de las exposiciones, es decir, a la persona que selecciona las piezas a exponer y establece la línea discursiva narrativa bajo la que se organizarán. Proviene de la expresión anglosajona curator.

3. Para corroborar si existía asociación entre estas variables se realizó un análisis chi cuadrado y un V de Crammer, que nos permitió comprobar su nivel de intensidad. Los resultados obtenidos señalaron que en todos los cruces realizados entre la variable dependiente y cada una de las variables independientes existe asociación, lo que quiere decir que los medios de comunicación utilizados (Facebook, Twitter, e-mail, web site, redes sociales y medios de comunicación propios) están relacionados con el volumen de visitantes registrados durante el último año.

4. Los enfoques cualitativos en estudios de aspectos relacionados con la gestión de museos han sido desarrollados en Argentina, por ejemplo, por María Viola (2013).

\section{Nota}

Este artículo está asociado al desarrollo del proyecto de investigación Fondecyt n. 1120313: Los museos y su público, evaluación y análisis de la comunicación y gestión de las audiencias museísticas en Chile.

\section{Bibliografía}

Alonso-Valero, Pilar; Alonso-Valero, Lucía; Caswell, Graham D. (2004). "Museos y medios de comunicación". Museo. Revista de la Asociación Profesional de Museólogos de España, n. 9, pp. 153-156.

http://dialnet.unirioja.es/servlet/dcfichero_articulo?codigo=2688120
Álvarez, Paula; Sebastián, Izascun (2010). “Una relación a tres: museos, medios de comunicación y públicos. Reflexiones y experiencias desde el departamento de comunicación del Musac". Mus-A: Revista de los museos de Andalucía, n. 12, pp. 76-84.

http://www.juntadeandalucia.es/culturaydeporte/museos/ media/docs/PORTAL_musa_n12_redc.pdf

Annis, Sheldon (1994). "The museum as a staging ground for symbolic action". En: Kavanagh, Gaynor (Ed.) Museum provision and professionalism. Routledge, pp. 21-25. ISBN: $041511280 \mathrm{X}$

Antinucci, Francesco (2004). Comunicare nel museo. Roma: Laterza. ISBN: 9788842072232

Bialogorski, Mirta; Cousillas, Ana-María (2008). "Una propuesta de evaluación innovadora en los estudios de visitantes de un museo de Buenos Aires". Mus-A: Revista de los museos de Andalucía, n. 10, pp. 98-101.

http://www.juntadeandalucia.es/culturaydeporte/museos/ media/docs/PORTAL_musa_10_ok.pdf

Bitgood, Stephen; Thompson, Donald (1987). "How do people perceive museums, parks, and zoos". Visitor behavior, v. 2, n. 3, pp. 9-10.

http://kora.matrix.msu.edu/files/31/173/1F-AD-146-8-VSAa0a2k7-a_5730.pdf

Caballero-Zoreda, Luis (1980). "El museo: funciones, personal y su formación". Boletín de la Anabad, v. 30, n. 3, pp. 377-385

http://dialnet.unirioja.es/servlet/fichero_articulo?codigo $=9$ 65418 \&orden $=67809$

Caballero-Zoreda, Luis (1986). "Del objeto al público: el informador en los museos". Boletín de la Anabad, v. 36, n. 1-2, pp. 259-280.

http://dialnet.unirioja.es/descarga/articulo/806419.pdf

Cóppola, Tatiana; González, Fernando (2009). Museos en el Uruguay: Una visión económica. Trabajo de investigación monográfico para la obtención del título de Licenciado en Economía. Universidad de la República. Facultad de Ciencias Económicas y de Administración.

http://www.ccee.edu.uy/investigacion/cultura/Museos_ en_el_Uruguay_Una_vision_economica.pdf

De-las-Nieves-Sarno, Alicia; Grandi, María-Emilia; Lloret, Florencia (2006). "Museos: ¿nuevos públicos o nuevas actitudes hacia sus públicos?”. Estudio de museología de Rosario. http://www.ilam.org/viejo/ILAMDOC/Museosnuevos.PDF

Fatás-Monforte, Pilar (2004). "Estrategias de comunicación de museos: el caso del Museo de Altamira". Museo: Revista de la Asociación Profesional de Museólogos de España, n. 9, pp. 131-149.

http://www.apme.es/revista/museo09_131.pdf

Jiménez-Losantos, Encarna (2004). "El museo se hace noticia”. Museo: Revista de la Asociación Profesional de Museólogos de España, n. 9, pp. 49-61.

http://www.apme.es/revista/museo09_049.pdf

Lledó, Joaquín (2003). "Los museos en los medios". Museo: Revista de la Asociación Profesional de Museólogos de Espa- 
ña, n. 9, pp. 43-46.

http://www.apme.es/revista/museo09_043.pdf

Martín-Guglielmino, Marcelo (2007). “La difusión del patrimonio. Actualización y debate". E-rph: Revista electrónica de patrimonio histórico, n. 1, pp. 195-216.

http://www.revistadepatrimonio.es/revistas/numero1/ difusion/estudios/articulo.php

Martínez-Peláez, Agustín; Oliva-Marañón, Carlos; Rodríguez-Rivas, Ana-María (2012) "Comunicación interna y externa en el Museo Reina Sofía: interacción del público en un entorno virtual". Telos: Cuadernos de comunicación e innovación, n. 90, pp. 71-78.

http://telos.fundaciontelefonica.com/seccion=1266\&idioma=es ES\&id=2012013116330001\&activo=6.do

Mateos-Rusillo, Santos (2008). "Hacia una comunicación global del patrimonio cultural, o cómo potenciar su uso fomentando su preservación". Mateos-Rusillo, Santos (coord.). La comunicación global del patrimonio cultural. Gijón: Trea, pp. 19-52. ISBN: 9788497043748

Mateos-Rusillo, Santos (2012). Manual de comunicación para museos y atractivos patrimoniales. Gijón: Ediciones Trea. ISBN: 8497046226

Meluch, Wendy (2010). "Survey \& focus groups". Visitors studies, n. 101. Documents of the American Association of Museums.

http://care-aam.org/documents/vs/Meluch_2010.pdf

Nardi, Emma (2010). "La mediazione culturale nei musei come forma narratologica". Cadmo, v. 18, n. 1, pp. 33-45.

http://goo.gl//zGE3kv

http://dx.doi.org/10.3280/CAD2010-001004

Oliveira, Andrea; Capriotti, Paul (2013). “Gestión estratégica de los públicos en museos. De la identificación a la comunicación". El profesional de la información, v. 22, n. 3, pp. 210-214.

http://www.kulturklik.euskadi.net/wp-content/ uploads/2013/10/E130202.pdf

http://dx.doi.org/10.3145/epi.2013.may.03

Pulido, Natividad (2004). "El museo es noticia". Museo: Revista de la Asociación Profesional de Museólogos de España, n. 9, pp.19-32.

http://www.apme.es/revista/museo09_019.pdf

Ruiz, María-Cristina; Aramburo, Felipe (2011). "Investigación cualitativa en museos. Métodos, técnicas y posibilidades. Una introducción". Revista museo lúdica, v. 14, n. 2627, pp. 22-23.

http://www.cienciayjuego.com/jhome/index.php?option=com_ content\&view $=$ article \&id=413:investigacion-cualitativaen-museos-metodos-tecnicas-y-posibilidades-unaintroduccion\&catid=52:22-23

Saavedra-Cruz, Francisco (2013). "Estudio exploratorio sobre el ámbito de la comunicación en los museos de la ciudad de Valparaíso". En: Simposio comunicación, museos y gestión de la cultura en tiempos de ciudadanos 2.0. III Congreso intl del conocimiento, Santiago de Chile.

Sanguinetti, María-del-Rosario; Garré, Fabián (2001). “Estudio de público: herramienta fundamental para el desarrollo de un proyecto de marketing de museos". Biblios: Revista electrónica de bibliotecología, archivología y museología, v. 3, n. 10, octubre-diciembre.

http://www.redalyc.org/articulo.oa?id=16110309

Styliani, Sylaiou; Fotis, Liarokapis; Kostas, Kotsakis; Petros, Patias (2009). "Virtual museums, a survey and some issues for consideration". Journal of cultural heritage, v. 10, n. 4, pp. 520-528.

http://dx.doi.org/10.1016/j.culher.2009.03.003

Viñaras-Abad, Mónica (2005). “Una aproximación a la gestión de la comunicación en los museos: cambios y tendencias en el cambio de siglo". Vivat academia, n. 67, pp. 37-63. http://www.vivatacademia.net/h/anteriores/n67/Num67/ PDFs/n67-3.pdf

Viñarás-Abad, Mónica (2008). "La gestión de la comunicación en los museos: auditoría de relaciones públicas". Questión, v. 1, n. 18.

http://perio.un/p.edu.ar/ojs/index.php/question/article/ view/587/498

Viola, María (2013). “Diversidad de estrategias en la gestión de museos". En: Simposio comunicación, museos y gestión de la cultura en tiempos de ciudadanos 2.0. III Congreso intl del conocimiento. Santiago de Chile.

\section{Suscripción EPI sólo online}

Pensando sobre todo en los posibles suscriptores latinoamericanos, ya no es obligatorio pagar la suscripción impresa de EPI para acceder a la online.

EPI se ofrece a instituciones en suscripción "sólo online" a un precio considerablemente más reducido $(101,65+21 \%$ IVA euros/año = 123 euros/año), puesto que en esta modalidad no hay que cubrir los gastos de imprenta ni de correo postal.

Para los suscriptores particulares, el precio de la sucripción "sólo online" es de $62+21 \%$ IVA euros/año $=75$ euros/año. 